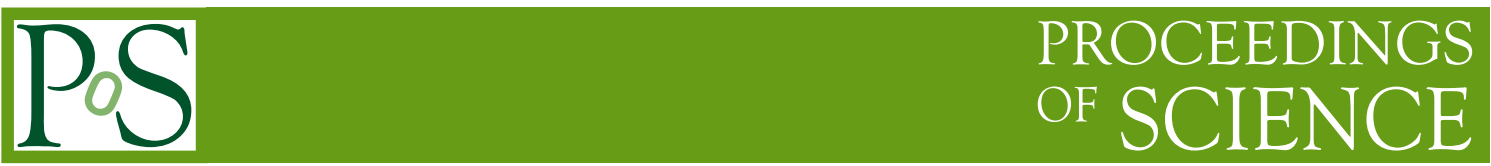

\title{
Beyond Standard Model Physics at LHeC and FCC-eh
}

\section{Georges Azuelos, ${ }^{a, b, *}$ Monica D'Onofrio, ${ }^{c}$ Oliver Fischer ${ }^{d}$ and the LHeC/FCC-eh Study Group}

${ }^{a}$ TRIUMF, Vancouver, BC, Canada

${ }^{b}$ Département de Physioque, Université de Montréal, Canada

${ }^{c}$ Oliver Lodge Laboratory, University of Liverpool, United Kingdom

${ }^{d}$ Department of Mathematical Sciences, University of Liverpool, United Kingdom

E-mail: georges.azuelos@umontreal.ca

\begin{abstract}
Thanks to the low background, negligible pileup and generally clean environement at ep colliders, a rich program of physics beyond the Standard Model (BSM) can be explored with precision measurements, complementing searches at LHC and future $p p$ colliders. We review some recent studies aimed at evaluating the reach and sensitivity of the next generation $e p$ colliders, the LHeC and FCC-eh, in probing a few BSM models, in particular compressed scenarios of supersymmetry, long-lived particles and dark sector, charged Higgs and leptoquarks.
\end{abstract}

40th International Conference on High Energy physics - ICHEP2020

July 28 - August 6, 2020

Prague, Czech Republic (virtual meeting)

\footnotetext{
${ }^{*}$ Speaker
} 


\section{Introduction}

Although limited by their center-of-mass energy, electron-proton colliders present some important characteristics which can be exploited in the search for Beyond-Standard-Model (BSM) physics: the absence of gluon-exchange, which is omnipresent at $p p$ colliders, implies very low backgrounds and nearly negligible pile-up; the possibility of polarized electron beams allows for detailed electroweak studies; parton distribution functions (PDF's) will be dramatically improved [1], allowing for much reduced theoretical uncertainties in searches for new physics. Given the lack of discovery, up to now, of BSM physics at high energy scales at $p p$ colliders, it becomes important to complement the search of new physics by precision measurements and by exploring challenging regions of parameter space at $e p$ colliders,

A conceptual design report (CDR) on the physics, machine and detector of a next-generation electron-proton collider was published in 2012 [2], and an updated version has very recently been released [3]. At the $\mathrm{LHeC}$, a $60 \mathrm{GeV}$ electron beam, produced in an energy-recovery Linac, collides with the $7 \mathrm{TeV}$ LHC proton beam. The center-of-mass $(\mathrm{cm})$ energy is $1.30 \mathrm{TeV}$. At the FCC-eh, the collision is with the $50 \mathrm{TeV}$ proton beam of the FCC-hh accelerator, and the cm energy is $3.5 \mathrm{TeV}$.

There is a rich program of BSM physics of which a few selected topics are discussed here, More information on the $\mathrm{LHeC} / \mathrm{FCC}$-eh physics, accelerator and detector, can be found in these proceedings.

\section{Supersymmetry}

In the search for electroweakinos, one region of parameter space which is not easily accessible at the LHC is in the so-called compressed scenario, where the NLSP, a chargino, neutralino or slepton, is close in mass to the LSP. This scenario is difficult to investigate at the LHC because the decay products are soft and not reconstructed, and the signal is kinematically similar to the SM background. At ep colliders, the production process is $e^{-} p \rightarrow e^{-} j \tilde{\chi} \tilde{\chi}$. A case study [4], based on BDT signal optimization and fast detector simulation with Delphes, assumes that $\tilde{\chi}^{0}$ is a Bino while $\tilde{\chi}_{1}^{ \pm} / \tilde{\chi}_{2}^{0}$ are Wino's, and that the mass difference between the $\tilde{\ell}$ and the $\tilde{\chi}_{1}^{ \pm} / \tilde{\chi}_{2}^{0}$ is $35 \mathrm{GeV}$. This allows the slepton to be produced on-shell. Assuming nearly degenerate $\tilde{\chi}_{1}^{ \pm}, \tilde{\chi}_{2}^{0}$ and the LSP, $\tilde{\chi}_{1}^{0}$, a limit of $\sim 450 \mathrm{GeV}$ on the mass of $\tilde{\chi}$ is obtained at FCC-eh with $1 \mathrm{ab}^{-1}$, as shown in Fig. 1 (left). This is comparable, or somewhat higher than what we can hope to obtain at HL-LHC $(\sim 400 \mathrm{GeV})$. In a decoupled scenario (a heavy slepton), the production cross section is lower and the limit at $\mathrm{LHeC}$ would be $\sim 2 \sigma$ [5] for a mass of $115 \mathrm{GeV}$.

High sensitivity of $e p$ colliders has also been shown for other scenarios of supersymmetry: in R-parity violating (RPV) resonant production of sbottom, $e^{-} p \rightarrow \tilde{b}^{*} \rightarrow \mu^{-} j$, for which present bounds $\lambda_{113}^{\prime}, \lambda_{123}^{\prime}<0.18$ and $\lambda_{231}^{\prime}, \lambda_{232}^{\prime}<0.45$, considerably improvement by an order of magnitude can be obtained with just $1 \mathrm{fb}^{-1}$ at the LHeC for low $\tilde{b}$ mass [6]. This can also be interpreted as a scalar LQ production and decay with generation mixing.

Another RPV study searches for prompt decay of low-mass gluinos, a region of parameter space called "stealth SUSY", for which the signal is composed of multiple soft jets, and which is therefore difficult to study at $p p$ colliders. A 95\% exclusion limit can be obtained at LHeC, with 1 $\mathrm{ab}^{-1}$ and $1 \%$ syst. error, for masses in the range $50-70 \mathrm{GeV}[7]$. 
It should be mentioned that, with the much improved PDF's obtained at LHeC, various LHC results can be re-interpreted and better mass limits obtained, such as for heavy gluinos $[1,8]$.
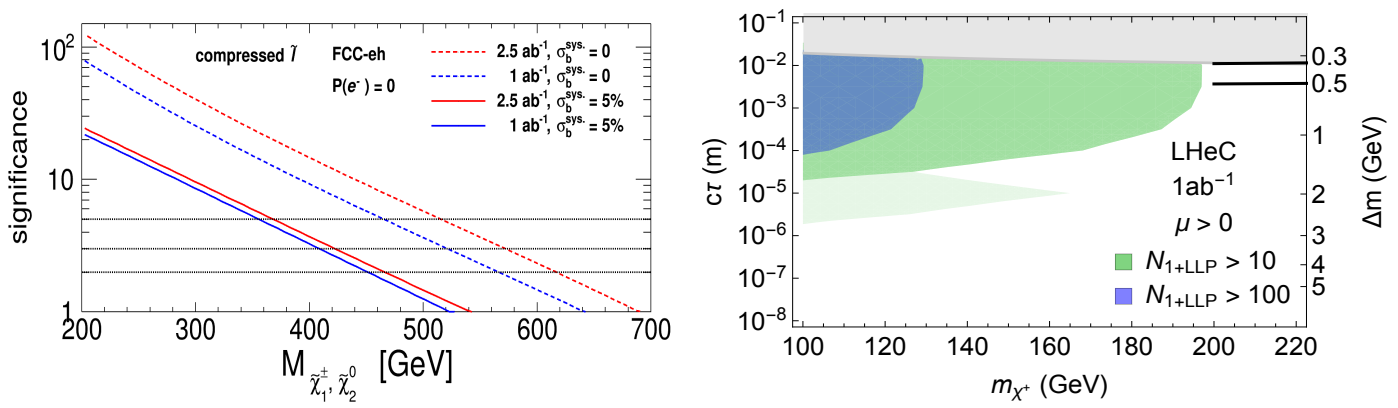

Figure 1: Left: Significance [4] as a function of the mass of $\tilde{\chi}_{1}^{ \pm}$and $\tilde{\chi}_{2}^{0}$ for the compressed slepton scenario at the FCC-eh with unpolarized beams and integrated luminosities of $1 \mathrm{ab}^{-1}$ and $2.5 \mathrm{ab}^{-1}$; Right: Exclusion limits on Higgsino masses as a function of their lifetime. Coloured regions denote where 10 or 100 events with at least one LLP decay are observed. For details, see [9].

\section{Long-Lived Particles and Dark Sector}

The clean environment and low pileup at $e p$ colliders also allow to search for long-lived particles (LLP) by looking for displaced vertices [9]. For example, for Higgsinos in compressed scenarios, small mixing and small mass splitting could imply a long lifetime. By looking for displaced vertices or a large impact parameter of a single low momentum track, one can be sensitive to the signal of their decay. It is difficult to predict how well the background can be controlled, but, assuming 5-16 $\mu \mathrm{m}$ tracking resolution and 50-400 MeV single track reconstruction, and depending on the number of single or double displaced vertices observed, a very significant improvement on the reach can be obtained at $\mathrm{LHeC}$ or FCC-eh over the limits expected from disappearing track searches at the HL-LHC, as shown in Fig. 1 (right). The search for LLP's can also apply to an analysis of light long-lived scalars with small mixing to the SM Higgs, yielding improved constraints on the $B R(H \rightarrow X X)[10]$.

Electron-proton colliders can be used to search for heavy neutrinos, $N$, in the process $e^{-} p \rightarrow$ $N^{-} j ; N^{-} \ell^{-} j j$ [11]. In particular, in low-scale see-saw models with two sterile neutrinos, the mixing with SM neutrinos is expected to be very weak, leading to a displaced vertex from the decay of the heavy neutrino. A search [12,13] for $e^{-} p \rightarrow \mu^{-}+3 j$, based on BDT optimization, assuming a tracking resolution of $8 \mu \mathrm{m}$ and a sensitivity of $40 \mu \mathrm{m}$ for the displacement of the vertex, shows that the constraint on the mixing angles $\Theta_{e \mu}^{2}$ and $\Theta_{e e}^{2}$ can be very significantly improved over current limits (Fig. 2, left). In a general effective Lagrangian model, sizable lepton-violating effects can be investigated [14] for couplings of $\mathrm{O}(1)$. It has also been shown [15] that one could discriminate between a scalar or vector effective interactions using polarized electrons. Also, in a Left-Right Symmetric model, a distinctive signature $e^{-} p \rightarrow e^{+} j$, due to the Majorana nature of the neutrino, can be investigated [16] in regions inaccessible by other searches, assuming good lepton-charge identification.

Dark photons arise from an additional $U(1)$ with weak kinematical mixing with hypercharge. Weak mixing and low mass imply decay to pairs of electrons, muons or light quarks, with long 

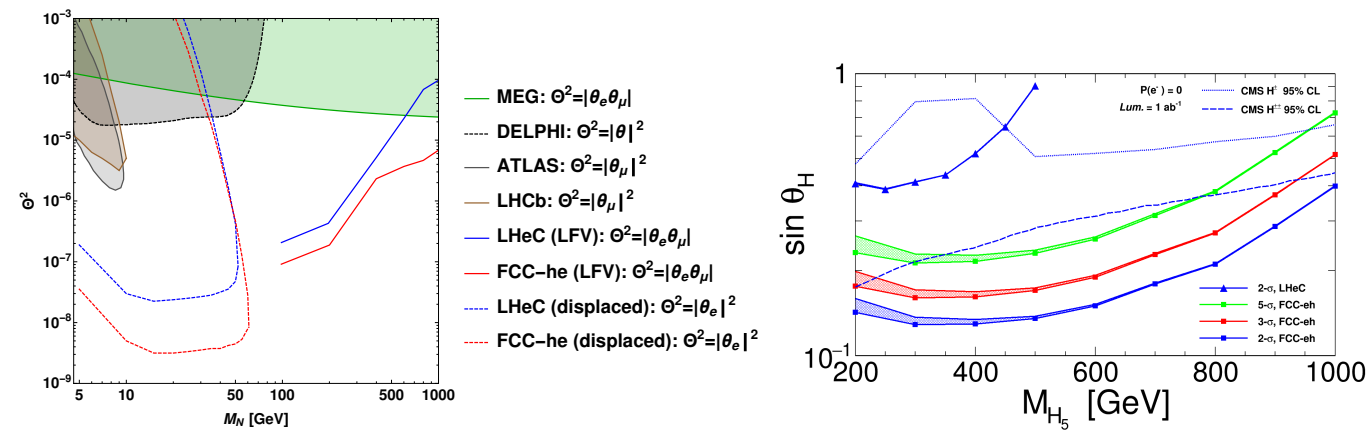

Figure 2: Left: Sensitivity of the LFV lepton-trijet searches and the displaced vertex searches (at $95 \%$ C.L.) [12] compared to the current exclusion limits from other colliders; Right: Limit Contours for the case of singly charged Higgs for FCC-eh and LHeC [21]. An unpolarized beam and a 10\% systematic uncertainty for background yields is assumed.

lifetime and consequently a displaced vertex. The excluded region of the mixing angle could be improved, depending on the background and assumed efficiency [17].

Axion-like particles (ALP's) are pseudoscalar particles which can act as a possible candidate mediator to the dark sector. Some unexplored parameter space can be covered at $e p$ colliders for not-so-low mass ALP's [18].

To set these results in context, in the briefing book of the European Strategy update [19] it is shown how e $p$ colliders can complement other searches for the dark sector: dark scalar mixing with the Higgs boson, axion-like particles, limits on spin-independent wimp-nucleon coupling, based on the Higgs portal model, with majorana or scalar DM.

\section{Charged Higgs}

As shown in [20], the measurement of the couplings of the SM Higgs can be performed with remarkable precision at the $\mathrm{LHeC}$ and FCC-eh. Here, as an example of an extended BSM Higgs sector, the Georgi-Machacek (GM) model, with higher isospin multiplets, built to satisfy explicitly custodial symmetry at tree level, is considered. It contains a bidoublet and two triplets. The physical states include a quintuplet which does not couple to fermions and which can therefore be produced only by vector boson fusion. There are two basic parameters: the mass and the coupling. An analysis [21] of the singly charged component of the Higgs quintuplet, using fast detector simulation and BDT signal optimization, predicts a 95\% sensitivity to coupling, as shown in Fig. 2 (right), to be compared with present limits from LHC (dashed curve). A similar analysis [22] has been performed for the doubly-charged Higgs of the GM model.

In the context of a 2-Higgs-doublet model (2HDM), type III, the charged Higgs, produced in the reaction $e^{-} p \rightarrow v H^{-} q$, can decay in a flavor-violating process into quarks. It has been shown [23] that a $3 \sigma$ limit can be obtained with $100 \mathrm{fb}^{-1}$ of integrated luminosity at LHeC, on the decay $H^{-} \rightarrow \bar{c} b$ for a Higgs mass of $100-200 \mathrm{GeV}$.

A search for a light charged Higgs of the NMSSM [24], containing an additional singlet $S$, decaying as $H^{-} \rightarrow \bar{c} s$ or $\bar{u} s$ can be constrained with $4.4 \sigma$, for a mass of $114 \mathrm{GeV}$, with $1 \mathrm{ab}^{-1}$ of 
integrated luminosity at FCC-eh.

\section{Leptoquarks}

Leptoquarks (LQ's) have both lepton and baryon quantum number and are predicted in various models of high energy physics. They are classified according to their representation in the SM gauge groups [25], and they can be scalar or vector in nature. In the last few years, they have gained in theoretical interest, as they have been proposed as a solution to the so-called B-anomalies: excesses or deficiencies observed in ratios of branching ratios between different generations, leading to question lepton universality. Some of these leptoquarks, in particular $S_{3}, R_{2}$ could explain these discrepancies with SM predictions [26]. The $\tilde{R}_{2}$ LQ can couple to right-handed neutrinos, which provides new production channels for the former and decay channels for the latter [27].

At the LHC, the main LQ production process is pair production by the strong interaction, which is independent of the coupling $L Q-e-q$. The present mass limits are therefore quite constraining. They are usually presented as a function of the branching ratio to charged lepton, which is, of course related to the various couplings. Nevertheless, some region of parameter space can still be explored at the $\mathrm{LHeC}$, when the $\mathrm{BR}$ is small, or at FCC-eh for masses greater than $\sim 1 \mathrm{TeV}$. If a LQ is discovered, very high sensitivity to the coupling can be reached at $e p$ colliders since they would be produced resonantly in the s-channel, allowing to extend the region which can be explored. The LQ properties could also be measured with high precision [2]: the fermion number, the charge, can be determined from the size and asymmetry in production from $e^{+}$and $e^{-}$. The chiral structure can also be studied with polarized beams. A scalar leptoquark decaying to a lepton and a quark has the signature of a R-parity violation of a squark decay and can therefore be re-interpreted as such. If the LQ mass is very high, much higher than the cm energy of the collider, the process is interpreted as a contact interaction.

\section{Conclusion}

Thanks to clean environment, low pileup, the possibility of precision measurements at $e p$ colliders offers a rich program of BSM physics, of which selected searches have been discussed here. The $\mathrm{LHeC}$ can probe sensitive regions of parameter space difficult of access at LHC and complement results from the LHC whereas the FCC-eh opens the higher energy regime.

\section{References}

[1] C. Gwenlan, contribution to these proceeedings

[2] J. L. Abelleira Fernandez et al. [LHeC Study Group], J. Phys. G 39 (2012), 075001

[3] P. Agostini et al. [LHeC and FCC-he Study Group], [arXiv:2007.14491 [hep-ex]].

[4] G. Azuelos, M. D’Onofrio, S. Iwamoto and K. Wang, Phys. Rev. D 101 (2020) no.9, 095015

[5] C. Han, R. Li, R. Q. Pan and K. Wang, Phys. Rev. D 98 (2018) no.11, 115003 
[6] S. Kuday, J. Korean Phys. Soc. 64 (2014), 1783-1787

[7] D. Curtin, K. Deshpande, O. Fischer and J. Zurita, Phys. Rev. D 99 (2019) no.5, 055011

[8] J. L. Abelleira Fernandez et al. [LHeC Study Group], [arXiv:1211.5102 [hep-ex]].

[9] D. Curtin, K. Deshpande, O. Fischer and J. Zurita, JHEP 07 (2018), 024

[10] K. Cheung, O. Fischer, Z. S. Wang and J. Zurita, [arXiv:2008.09614 [hep-ph]].

[11] S. Antusch, E. Cazzato and O. Fischer, Int. J. Mod. Phys. A 32 (2017) no.14, 1750078

[12] S. Antusch, O. Fischer and A. Hammad, JHEP 03 (2020), 110

[13] S. Mondal and S. K. Rai, Phys. Rev. D 94 (2016) no.3, 033008

[14] L. Duarte, G. A. González-Sprinberg and O. A. Sampayo, Phys. Rev. D 91 (2015) no.5, 053007

[15] L. Duarte, G. Zapata and O. A. Sampayo, Eur. Phys. J. C 78 (2018) no.5, 352

[16] M. Lindner, F. S. Queiroz, W. Rodejohann and C. E. Yaguna, JHEP 06 (2016), 140

[17] M. D’Onofrio, O. Fischer and Z. S. Wang, Phys. Rev. D 101 (2020) no.1, 015020

[18] C. X. Yue, M. Z. Liu and Y. C. Guo, Phys. Rev. D 100 (2019) no.1, 015020

[19] J. Ellis, M. A. G. Garcia, N. Nagata, D. V. Nanopoulos and K. A. Olive, JCAP 01 (2020), 035

[20] U. Klein, contribution to these proceedings

[21] G. Azuelos, H. Sun and K. Wang, Phys. Rev. D 97 (2018) no.11, 116005

[22] H. Sun, X. Luo, W. Wei and T. Liu, Phys. Rev. D 96 (2017) no.9, 095003

[23] J. Hernández-Sánchez, O. Flores-Sánchez, C. G. Honorato, S. Moretti and S. Rosado, PoS CHARGED2016 (2017), 032

[24] S. P. Das, J. Hernandez-Sanchez, S. Moretti and A. Rosado, [arXiv:1806.08361 [hep-ph]].

[25] I. Doršner, S. Fajfer, A. Greljo, J. F. Kamenik and N. Košnik, Phys. Rept. 641 (2016), 1-68

[26] A. Angelescu, D. Bečirević, D. A. Faroughy and O. Sumensari, JHEP 10 (2018), 183

[27] S. Mandal, M. Mitra and N. Sinha, Phys. Rev. D 98 (2018) no.9, 095004 